\title{
Radio resource management for user-relay assisted OFDMA-based wireless networks
}

\author{
İlhan Baştürk*, Berna Özbek \\ Electrical and Electronics Engineering Department, Izmir Institute of Technology, Urla 35430, Izmir, Turkey
}

\section{A R T I C L E I N F O}

\section{Article history:}

Received 6 February 2015

Accepted 27 January 2016

\section{Keywords:}

User-relaying

Radio resource management

OFDMA

Relay selection

\begin{abstract}
A B S T R A C T
Orthogonal frequency division multiple access (OFDMA) based relay assisted networks are important for current and next-generation wireless networks since they have great potential to provide high data rate to users at anywhere, anytime. Towards that end, fixed relay assisted OFDMA networks are commonly utilized by the operators. However, they require supplementary costly infrastructure. User-relay assisted OFDMA-based networks are promising candidates to fulfill the demanding coverage and capacity requirements of future wireless networks in a cost efficient way. Thus, they have become popular as a complementary solution to fixed relay networks. Generic frame structure that divides the resource allocation frame into two subframes is mostly used in the literature for fixed and user-relay assisted networks in which it is assumed that a user can act as a destination and a relay simultaneously. However, it may be difficult to apply this assumption to practical systems because of the limitations of the current transceiver design. Thus, we propose a novel frame structure for user-relay assisted OFDMA-based wireless networks in order to cope with these drawbacks. The efficient radio resource management algorithms including relay selection and resource allocation are developed in order to exploit the opportunities of the proposed frame structure.
\end{abstract}

(c) 2016 Elsevier GmbH. All rights reserved.

\section{Introduction}

One of the expectations for the next-generation wireless communication networks is to provide ubiquitous high data rate coverage. OFDMA is one of the key technologies to achieve this objective due to its inherent robustness against frequency-selective fading and its capacity for achieving high spectral efficiency [1]. Conventional cellular networks are limited in their coverage and capacity. One of the solutions is to deploy more base stations (BSs) in order to overcome these limitations. However, this solution is not cost efficient for the service providers. An alternative cost efficient solution is the usage of a relay station (RS) as a third type of stations in a cellular network to construct a relay network consisting of BSs, RSs and Mobile Stations (MSs). The combination of OFDMA and relaying is one of the key technologies to deliver the promise of the reliable and high-data-rate coverage in the most cost effective manner.

Relaying strategies are mainly studied for two different purposes in wireless communication systems. One of them is called

\footnotetext{
* Corresponding author.

E-mail addresses: ilhan.basturk@adu.edu.tr (i. Baştürk), bernaozbek@iyte.edu.tr (B. Özbek).
}

cooperative diversity relaying mitigating signal power fluctuation due to multipath fading [2-6], and the other is called multi-hop relaying mitigating signal attenuation due to path-loss [7-10].

Relay assisted network architectures can be classified as fixed relay networks and mobile relay networks according to the movement of the relays [8-13]. In fixed relay networks, the relay stations are part of the network infrastructure, therefore their deployment will be an integral part of the network planning, design and deployment process. Fixed relay networks have been extensively studied in the literature and have already been included in the 4G Long Term Evolution (LTE)-Advanced standard [14]. In mobile relay networks, relay stations are not part of the fixed wireless infrastructure and their locations are not deterministic. The usage of mobile relay networks extends the coverage and increases the throughput $[10,15,16]$. Different types of mobile relays can be addressed as dedicated mobile relays that are mounted on moving vehicles and user terminals acting as mobile relays which are also called as user-relays. User-relaying is foreseen as one of the emerging technologies that will change and define the fifth generation (5G) telecommunication standard $[17,18]$. As the number of fixed RSs in a network is limited, the relay usability in the network is also limited. User-relays have more degrees of freedom compared to fixed RSs since the number of user-relays scales with the number of mobile users. The lower deployment cost is the main advantage 
of the user-relays since it is not required to add any costly infrastructure for relaying. Users' density which is a critical parameter for the relaying opportunity and the battery life are the challenges of user-relay assisted networks.

In order to fully realize the capacity and coverage benefits in relay assisted networks, efficient radio resource management (RRM) including relay selection and resource allocation (RA) is critical. The problem formulation may differ significantly in optimization objectives (rate maximization, power minimization), optimization constraints (fairness, load balancing), relaying protocols (amplify and forward (AF), decode and forward (DF)), relaying modes (full-duplex, half-duplex), relay types (fixed, mobile), antenna numbers of the source, destination and relay (single antenna/multiple antennas) and system architectures (downlink/uplink, single-cell/multi-cell). The RRM problem has been examined in numerous research works for OFDMA-based fixed relay networks [19-29]. The opportunities and challenges of the OFDMA-based fixed relay networks have been discussed in detail in $[19,20]$. In [21], RRM problem for sum rate maximization has been examined for single-cell OFDMA system. The problem has been solved optimally by using a continuous relaxation, since the problem belonging to the class of integer programming is very difficult to solve easily in direct forms. In [22], a low complexity heuristic solution has been presented for the same RRM problem. The fairness issue has been considered in [23-25] contrary to given studies earlier and heuristic RRM algorithms have been presented. In [26,27], the joint relay selection and RA problem have been investigated for the uplink single-cell OFDMA-based system. In [28,29], the RRM problem has been adapted for the multi-cell scenario. Moreover, multiple antennas are deployed at sources, relays, and destinations in $[30,31]$ to increase the system capacity. Efficient relay and antenna selection algorithms are developed in this multirelay and multi-antenna environment to fully exploit the benefits of the combination of fixed-relaying and multiple antennas technology. The works in [32-38] have examined the RRM problem for OFDMA-based mobile relay networks in which users act as relays. In [32], the RA strategy in OFDMA systems using an AF scheme has been considered, the optimal and suboptimal solutions for the power minimization have been presented. The fairness issue has been taken into consideration with subcarrier and power allocation schemes for uplink OFDMA in [33]. In [34], the joint optimization of relay selection, relay-strategy selection as AF or DF and RA have been considered to maximize an utility function for cellular networks. In [35], an optimal cooperation strategy has been developed for two-user uplink OFDMA systems, which have achieved the capacity region upper bound for DF scheme. In [34,35], it has been assumed that nodes are able to transmit and receive simultaneously on adjacent subcarriers. The feasibility of this assumption has been examined in [36] and a performance degradation has been observed since the orthogonality between different subcarriers is lost in practical communication systems. In [37,38], we have proposed suboptimal practical RRM solutions for the userrelay assisted OFDMA networks. In [37], heterogeneous networks which contain both macro BSs and access points have been combined with the mobile relaying technology and the efficient relay selection and the network interface selection have been presented for such a complex environment. In [38], a joint relay selection and RA algorithm has been examined by considering the queue information for each user. All these studies for fixed and mobile relay networks use a generic frame structure in which the downlink frame is partitioned into two consecutive subframes.

In this paper, we focus on the user-relay assisted OFDMA networks since relaying through users' terminal provides more flexibility to the cellular network by increasing the number of relay candidates and reducing the infrastructure cost. While fixed terminal relaying brings improvements in cellular systems, the implementation of user relaying will bring a huge gain to $5 \mathrm{G}$ based wireless networks employing different scenarios [17]. One of the scenarios examined in 5G based systems is device relaying with operator controlled link establishment corresponding to the framework which is considered in this paper. We propose a novel frame structure with effective relay selection and resource allocation for user-relay assisted OFDMA-based wireless networks. The contribution and features of the proposed scheme are summarized as follows:

- While keeping the same transmission time, we propose to divide the whole frame duration into three subframes instead of two subframes in order to classify the users as satisfied and unsatisfied users after an initial direct transmission session.

- The satisfied users are given permission to act as a relay and they are assigned as the user-relay candidates of the unsatisfied users.

- The users are not allowed to act as a destination and a relay simultaneously contrary to [34,35]. This assumption in which users are able to transmit and receive at the same time through adjacent subcarriers may have practical limitations with the current transceiver design [36].

The rest of this paper is organized as follows. In Section 2, we give the system model and assumptions. In Section 3, we examine the RRM strategy for user-relay assisted OFDMA networks with the proposed frame structure. The extensive performance evaluations are provided in Section 4. Finally, the conclusion is given in Section 5.

\section{System model}

We consider a single-cell downlink user relay-assisted OFDMAbased network topology as illustrated in Fig. 1. The BS is located in the center of the cell and $K$ users are distributed uniformly in the cell. The BS and each of the user which will be called as User Equipment (UE) in the rest of the paper have single antenna. The UEs can communicate to the BS either directly or through a userrelay.

The achievable performance and the required signalling of an relay selection scheme depend on the number of relay candidates. If all the available users are utilized as user-relay candidates as in $[10,32,34,39-43]$ this will cause maximum signalling overhead. The number of relay candidates, and thus signalling, can be significantly reduced by selecting the user-relays in a limited area around each

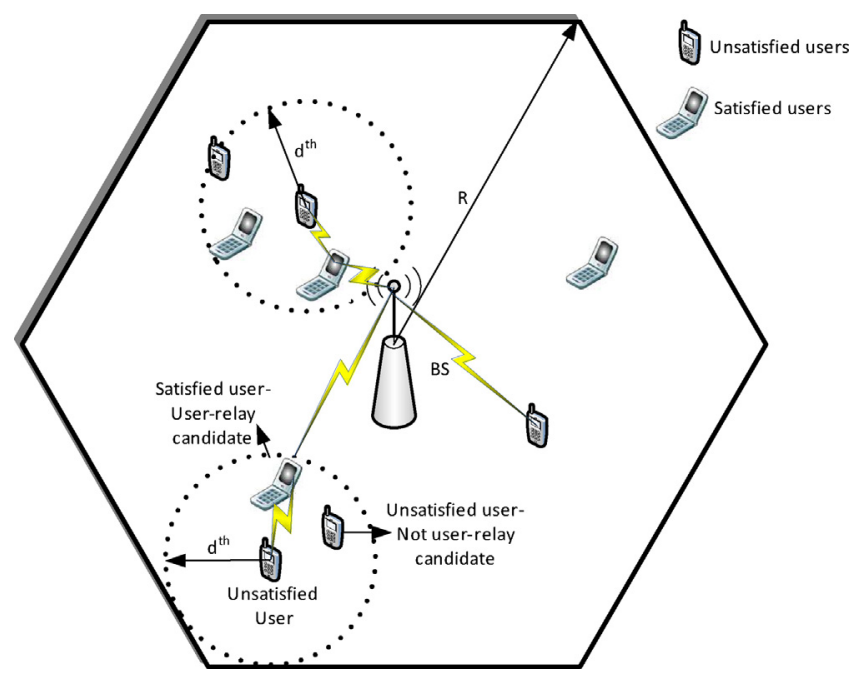

Fig. 1. User-relay assisted OFDMA network topology. 
user $[37,38,44,45]$. Thus, in this study, the users' relay candidates are selected by using the area whose radius is $d^{\text {th }}$ as shown in Fig. 1.

The DF relaying, half-duplex mode, and two-hop relaying are considered in this work. The total available bandwidth, $B$, is divided into $N$ subchannels and each subchannel consists of a set of adjacent Orthogonal Frequency Division Multiplexing (OFDM) subcarriers. The achievable data rate between two nodes is calculated for each subchannel as,

$R_{i \rightarrow j, n}=\frac{B}{N} \log _{2}\left(1+\gamma_{i \rightarrow j, n}\right)$

where $\gamma_{i \rightarrow j, n}=\frac{P_{i, n}\left|H_{i \rightarrow j, n}\right|^{2}}{N_{0}(B / N)}$ is the Signal to Noise Ratio(SNR) between a transmitter node $i$ and receiver node $j$ on subchannel $n . P_{i, n}$ is the transmitted power per subchannel for node $i . H_{i \rightarrow j, n}$ is the channel coefficient between node $i$ and node $j$ that includes path-loss, shadowing and fading. $N_{0}$ is the noise spectral density. The total transmission power is equally shared among all subchannels.

We assume that all required channel gains among BS and all UEs, and among UEs and user-relays are available perfectly at the BS where RA algorithms are performed as in user-relay assisted OFDMA systems [32-38] as well as fixed-relay assisted OFDMA systems [19-29]. The communication links between BS and userrelays and user-relays and UEs use the same subchannel at different time slots as in $[32,37,38]$. The relaying operation can also be performed by using different frequency bands [35,46], but computational complexity of the optimization increases considerably. Each user has its own threshold data rate, $R_{k}^{\text {th }}$, and the user which reaches its threshold data rate is called as satisfied user and the user that does not reach this rate is called as unsatisfied user as seen in Fig. 1.

\section{The proposed frame structure and RRM for user-relay assisted networks}

In this paper, we propose a novel frame structure that divides the downlink allocation frame into three subframes while keeping the same transmission duration as shown in Fig. 2. Our main motivation is to prevent the users to simultaneously act as a destination and a relay contrary to generic frame structure. The proposed scheme is more practical with current transceiver design, since any user does not have to receive its own data and to transmit the other users' data at the same time. A user has to meet its data rate requirement to be a relay candidate for other users.

In the first subframe with duration $T_{11}$, only direct communication between BS and UEs (all users) is allowed and this is indicated as $\mathrm{BS} \rightarrow \mathrm{UE}$. In the second subframe with duration $T_{12}$, BS sends data to UEs (unsatisfied users) and RSs (satisfied users) which are indicated as BS $\rightarrow$ UE and BS $\rightarrow$ RS, respectively. In the third subframe with duration $T_{13}$, UEs (unsatisfied users) receive information from RSs (satisfied users) and BS as labeled by BS $\rightarrow$ UE and RS $\rightarrow$ UE. Each subframe can contain more than one time slot according to

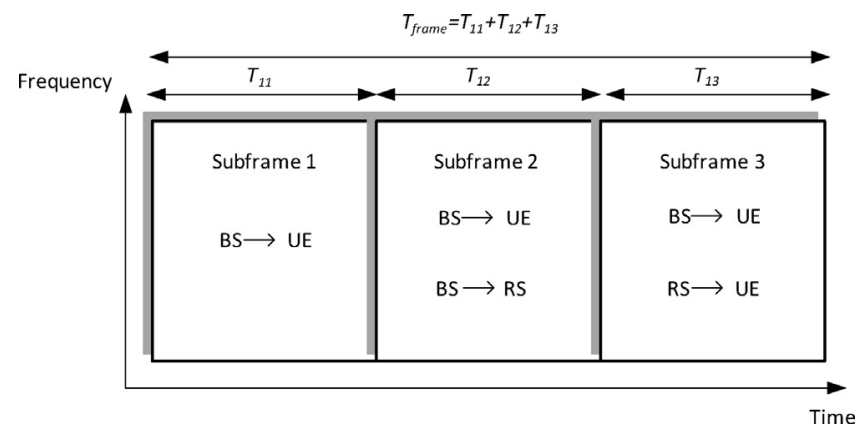

Fig. 2. The proposed frame structure. the subframe durations. Therefore, the number of resources allocated to the users are related to these durations. Higher subframe durations lead to higher number of OFDM symbols.

Efficient RRM algorithms are required to reveal the advantages of the proposed frame structure. The relay selection and RA are performed at the BS in the proposed model. At the beginning of an allocation frame, the BS has the related information belonging to each user about the possible user-relay candidates set which is constructed as follows:

$\mathbb{M}_{k}=\left\{r \mid d_{k \rightarrow r} \leq d^{t h} ; \quad r=1,2, \ldots, K, r \neq k\right\}, \quad k=1,2, \ldots, K$

where $d_{k \rightarrow r}$ is the distance between user $k$ and user-relay candidate $r$. The UEs can have the knowledge of their distance to the $\mathrm{BS}$ and to the other users, via the use of global positioning system $[45,47]$.

The RA is performed for all UEs by allowing only direct links through the duration $T_{11}$ at subframe 1 . At the end of the RA for subframe 1 , the users are classified as satisfied and unsatisfied. After this subframe, since the satisfied users will be in inactive mode, these users are assigned as the user-relay candidates for the unsatisfied users.

The relay selection and RA for the proposed frame structure is described in the following:

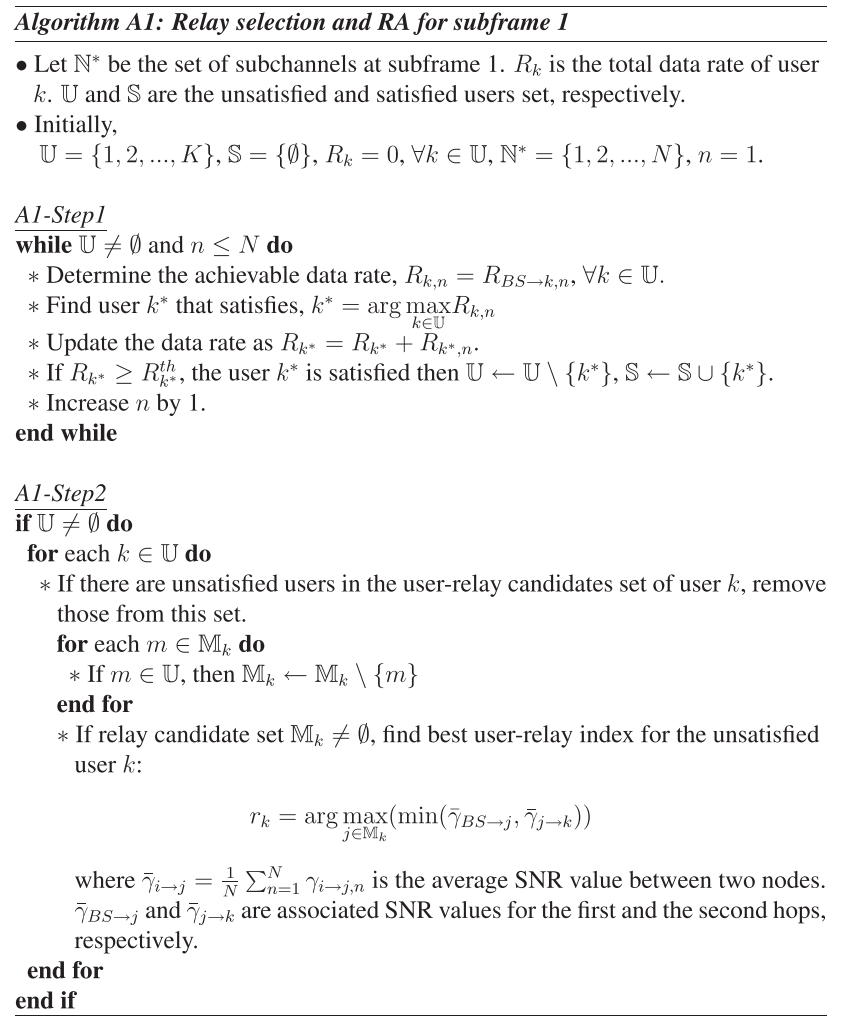

In the first step of the proposed Algorithm A1, the RA is performed for the subframe 1 in which the users communicate to BS directly. Each subchannel is allocated to the user that has the maximum achievable rate. The users admit subchannels until they fulfill their own data rate requirements. The total data rate of a user $k$ is defined as $R_{k}$ which is the sum of the achievable data rates of each subchannel allocated to this user. Thus, the number of subchannels allocated to each user effects the value of its total data rate. This step is terminated when all subchannels are allocated or unsatisfied users' set is empty. If the set of unsatisfied users is not empty, 
the second step of the algorithm is performed. In this step, the user-relay candidates sets, $\mathbb{M}_{k}$ which are determined at the beginning of this frame are updated. The unsatisfied users are removed from these sets, since only satisfied users can be assigned as userrelays. Then, the user-relay of any unsatisfied user is selected through its updated user-relay candidate set by using the average SNR values of the first and second hop links. Therefore, the unsatisfied users can communicate with BS not only directly but also through a user-relay.

The RA is performed by the BS for the second and third subframes as in Algorithm A2 given below.

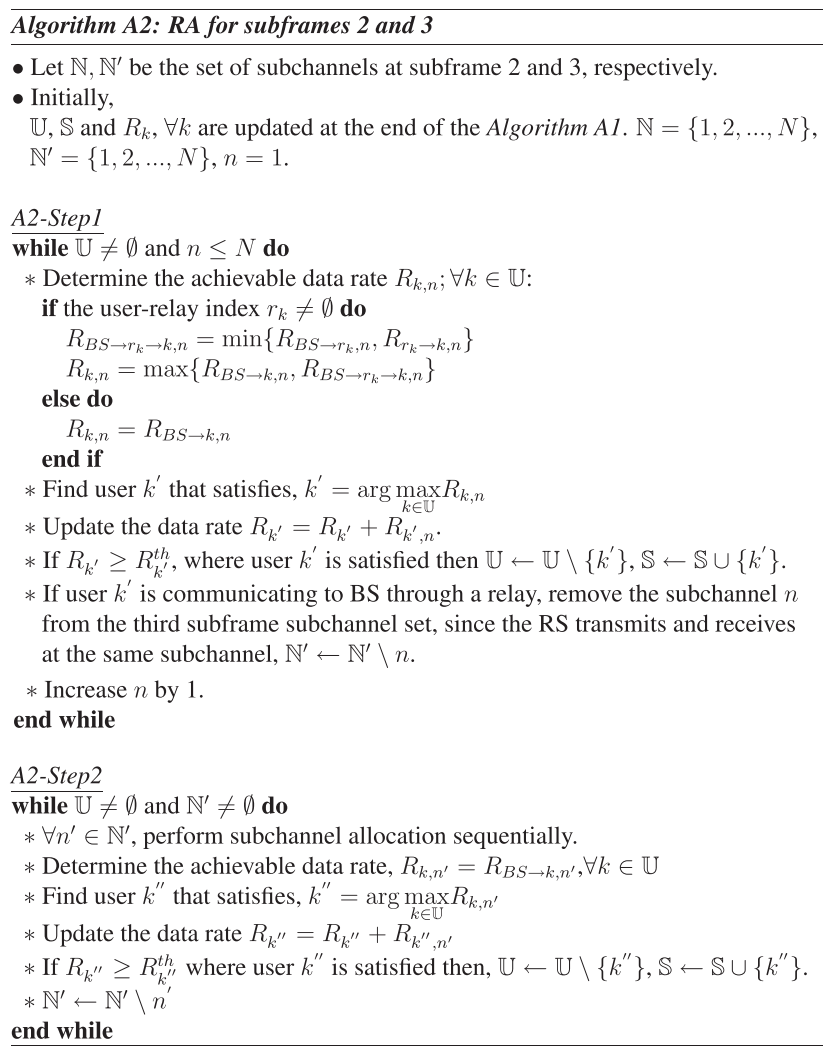

The Algorithm A2 is a two step RA algorithm. In the first step, the subchannels are allocated to the users which communicate to the BS directly and to the relayed users which communicate to the BS through a user-relay. In the second step, the subchannels are only allocated to the users that communicate to the BS directly. In the algorithm, the user-relays receive and transmit the data using the same subchannel in two consecutive subframes. If a subchannel is allocated to a relayed user in A2-Step 1, the same subchannel will also be reserved for the same user in A2Step 2. These subchannels are not used in A2-Step 2. The algorithm is terminated, when all users are satisfied or all subchannels are exhausted.

We illustrate the steps of the algorithm for the proposed frame structure in Fig. 3. We have 4 users labelled as UE1, UE2, UE3 and UE4. In the first subframe, the subchannels are allocated to the direct users by using A1-Step1. At the end of this subframe, the users are classified as satisfied and unsatisfied users according to their data rate requirements. We propose to use the satisfied users as the user-relay candidates of the unsatisfied users. Thus, the relay selection procedure A1-Step2 is performed and the relay candidates of the unsatisfied users are determined. In the example described in Fig. 3, UE1 and UE2 are the satisfied and UE3 and

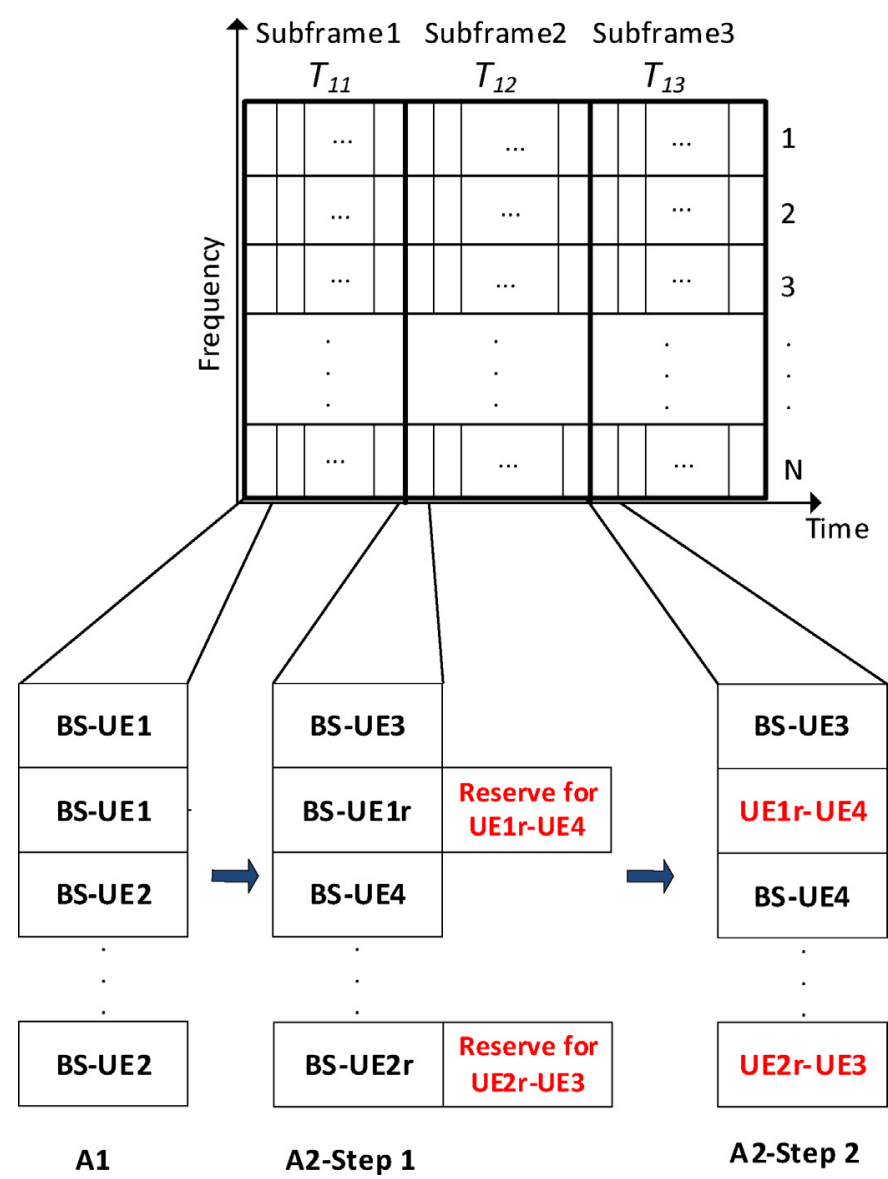

Fig. 3. Illustration of the RRM algorithms for the proposed frame structure.

UE4 are the unsatisfied users, respectively. Using the A1-Step2, it is determined that UE1 is the relay candidate of UE4, and UE2 is the relay candidate of UE3. In A2-Step1, the subchannel 1 and subchannel 3 in subframe 2 are allocated to the UE3 and UE4 for direct communication, respectively. The subchannel 2 and subchannel $\mathrm{N}$ in subframe 2 are allocated to the user-relays UE1r (UE1 acts as user-relay of UE4) and UE2r (UE2 acts as user-relay of UE3), respectively. In this step, we consider not only the first hop link between BS and user-relay but also the second hop link between user-relay and user for the data rate calculation of the relayed users. The data rates of the UE3 and UE4 with the help of relay is calculated as $R_{B S \rightarrow U E 2 r \rightarrow U E 3, n}=\min \left\{R_{B S \rightarrow U E 2 r, n}, R_{U E 2 r \rightarrow U E 3, n}\right\}$ and $R_{B S \rightarrow U E 1 r \rightarrow U E 4, n}=\min \left\{R_{B S \rightarrow U E 1 r, n}, R_{U E 1 r \rightarrow U E 4, n}\right\}$. The user-relays (UE1 $r$ and UE2r) receive and transmit the data using the same subchannel in two consecutive subframes (subframe 2 and subframe 3 ) as mentioned above. Thus, the subchannels 2 and $N$ in subframe 3 are reserved for the communication between UE1r $\rightarrow$ UE4 and $\mathrm{UE} 2 \mathrm{r} \rightarrow \mathrm{UE} 3$, respectively. Finally, in A2-Step2, the remaining subchannels in subframe 3 (the subchannel 1 and subchannel 3 that are not reserved for relaying) are allocated to the unsatisfied users for direct communication. Thus, the subchannel 1 and the subchannel 3 are allocated to the UE3 and UE4, respectively.

As mentioned before, each subframe can have more than one time slot depending on the subframe duration. In the RA algorithms, the time slot index is omitted since the procedure is the same for all time slots in one subframe at each step.

\section{Performance evaluations}

The performance evaluation of the proposed frame structure with efficient RRM algorithms are presented for user-relay assisted 
Table 1

Simulation parameters.

\begin{tabular}{ll}
\hline Parameter & Value \\
\hline Frequency & $2 \mathrm{GHz}$ \\
Bandwidth & $20 \mathrm{MHz}$ \\
Thermal noise density & $-134.89 \mathrm{dBm} / \mathrm{Hz}$ \\
BS Tx power & $46 \mathrm{dBm}$ \\
Fixed relay Tx power & $37 \mathrm{dBm}$ \\
User-relay Tx power & $23 \mathrm{dBm}$ \\
Cell radius $(R)$ & $750 \mathrm{~m}$ \\
UE min. close-in distance to BS & $35 \mathrm{~m}$ \\
Frame duration $\left(T_{\text {frame }}\right)$ & $10 \mathrm{~ms}$ \\
Path-loss model & $\mathrm{BS} \rightarrow \mathrm{UE}, \mathrm{BS} \rightarrow \mathrm{RS}$ and RS $\rightarrow$ UE \\
& $128.1+37.6 \log 10 \mathrm{~d}(\mathrm{~km})$ \\
Shadowing model & Lognormal distribution, \\
& $\mu=0, \sigma=10 \mathrm{~dB}$ \\
Multipath model & Extended Pedestrian A $(\mathrm{EPA})$ \\
\hline
\end{tabular}

OFDMA networks. We obtain the simulation results by using the parameters summarized in Table 1 . We consider a single cell network topology with a BS located in the center and surrounded by uniformly distributed UEs as seen in Fig. 4. In the simulations, not only the BS but also the UEs have single transmit antenna. Simulation results are performed for 1000 Monte-Carlo trials.

We compare four different OFDMA-based schemes in terms of percentage of satisfied users in order to reveal the advantages of the proposed frame structure in user-relay assisted networks. In the first scheme that is called $w / o$ relay, there is no relay in the system and all users communicate to the BS directly during the whole transmission frame. In other words, with duration $T_{\text {frame}}$, only direct communication between BS and UEs (BS $\rightarrow$ UE) is allowed. In this scheme, the RA is performed at BS by using a greedy algorithm that assigns the available resources to the users sequentially based on their channel gains until their threshold data rates are satisfied. Firstly, each subchannel is allocated to the link with the user having maximum channel gain. Then, the total data rate of the selected user is calculated and compared with the threshold data rate in order to determine if it is satisfied or not. The satisfied user is removed from the unallocated user set and the other subchannels are allocated to the remaining users until they are all satisfied or all the subchannels are exhausted.

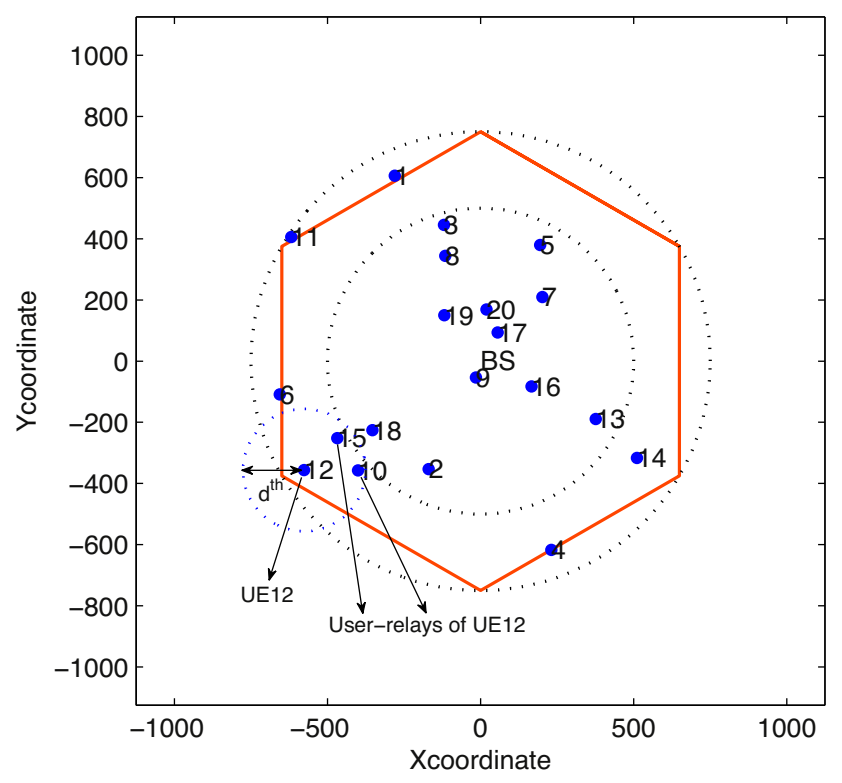

Fig. 4. User-relay assisted network topology.

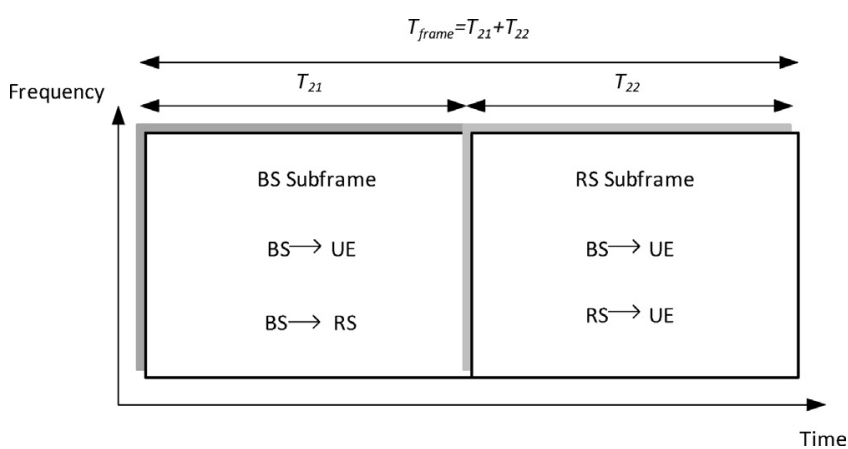

Fig. 5. Generic frame structure.

In the second and third schemes, generic frame structure that includes two consecutive subframes as illustrated in Fig. 5 is used for user-relay and fixed relay assisted networks, respectively. The subframe durations $T_{21}$ and $T_{22}$ are selected equal in this study. In the first subframe called BS subframe, BS transmits data to UEs (all users) and RSs (fixed or user-relays). In the second subframe called RS subframe, UEs (all users) receive data from BS and RSs (fixed or user relays). In this frame structure, the relay selection is performed at the BS by using path-loss of the first (between BS to candidate relay) and the second (between candidate relay to relayed user) hops. Firstly, the maximum path-loss value between the first and second hop link is determined for each user. Then, the candidate relay which has minimum value among them is selected as explained in [48]. The available resources are sequentially allocated to the users based on their channel gains in order to satisfy all users' data rate requirements. A subchannel is allocated to a user that has the maximum end to end link gain which is the direct link gain between the BS and the users for the direct communication and the minimum of the first and the second hop links' gain for the relayed users.

In the third scheme in which fixed relays are used, we locate 6 fixed relays at equal angles and equal distance to the BS as illustrated in Fig. 6. The distance of each fixed relay from the BS is chosen as $(2 R / 3)$ where $R$ is the radius of the cell. The BS $\rightarrow$ RS links have lognormal shadowing with $\sigma=4 \mathrm{~dB}$ and these links experience Rician fading with a Rician factor of $10 \mathrm{~dB}$.

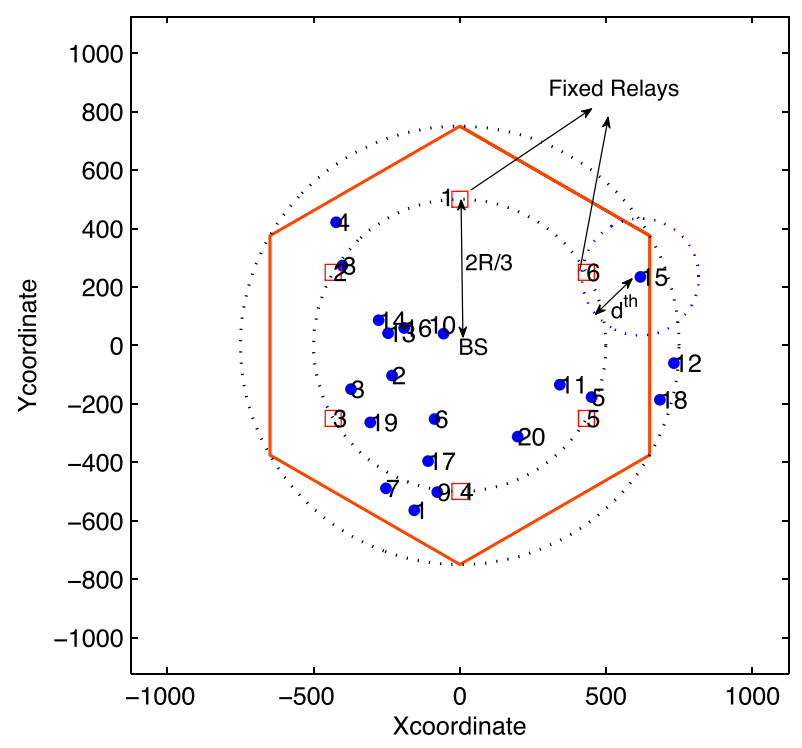

Fig. 6. Fixed-relay assisted network topology. 


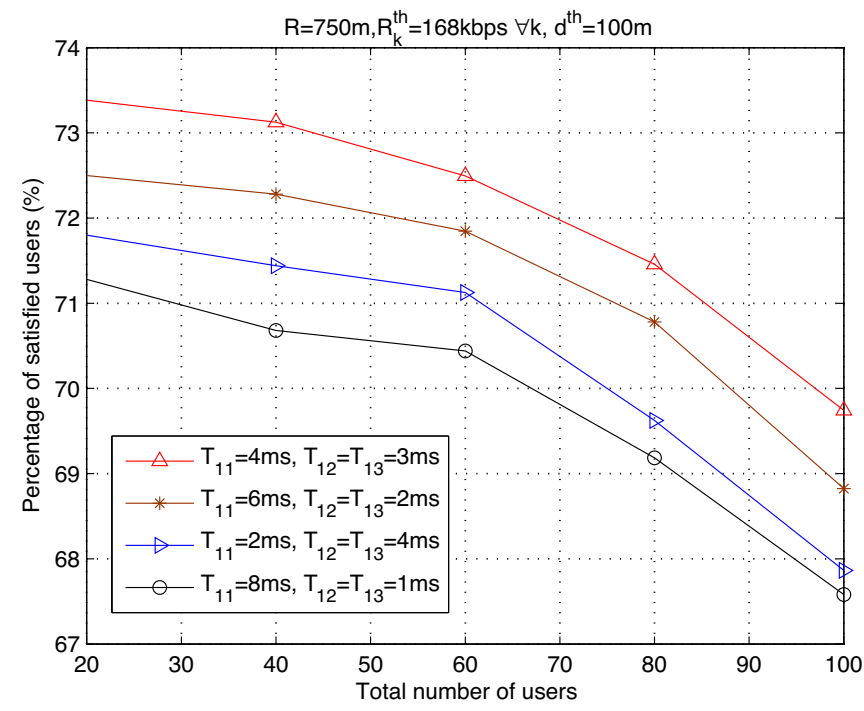

Fig. 7. Effect of the subframe durations on the percentage of satisfied users for $R_{k}^{\text {th }}=$ $168 \mathrm{kbps}$.

Finally, in the fourth scheme, the proposed frame structure which have three subframes with duration $T_{11}, T_{12}$ and $T_{13}$ as explained in Section 3 is used for user-relay assisted networks. Firstly, the durations of these subframes are determined before comparing the proposed frame structure with the other schemes. In this paper, we assume that the second and third subframe duration are equal as in the generic frame structure in order to decrease the complexity of the system. Thus, there are four options for the duration of subframe $1, T_{11}$ as $2 \mathrm{~ms}, 4 \mathrm{~ms}, 6 \mathrm{~ms}$ or $8 \mathrm{~ms}$ since the total frame duration, $T_{\text {frame }}$ is chosen as $10 \mathrm{~ms}$ for RA. The percentage of the satisfied users are obtained for various $T_{11}$ values. The simulation results are obtained for not only low but also high threshold data rate values as shown in Figs. 7 and 8, respectively. It is observed that the selection of $T_{11}$ as $4 \mathrm{~ms}$ gives the best performance on the percentage of satisfied users. Moreover, the number of user-relay candidates belonging to different subframe durations are obtained for low and high data rate threshold values of users in

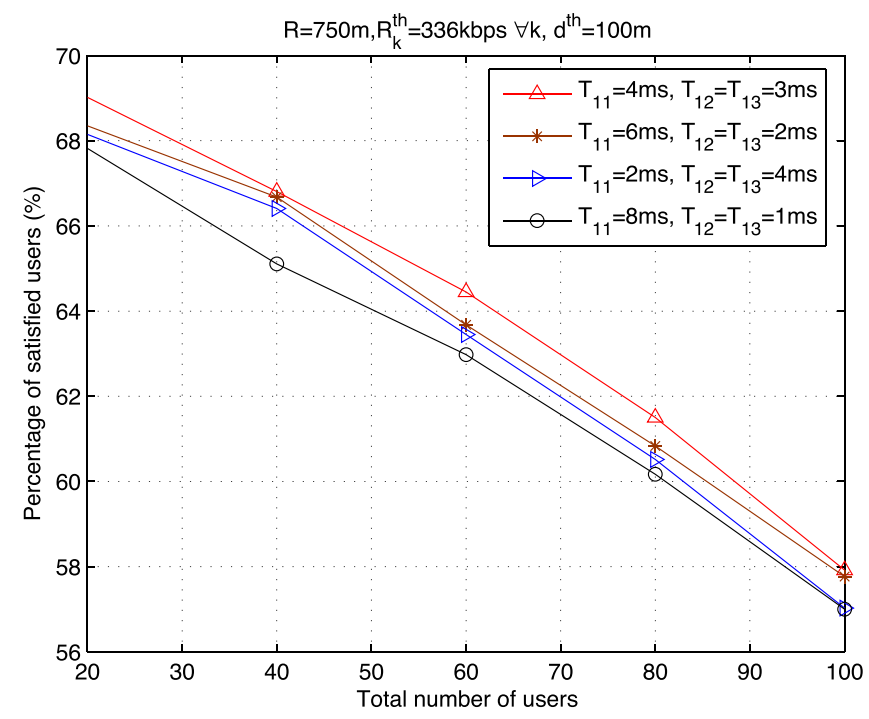

Fig. 8. Effect of the duration of subframes on the percentage of satisfied users for $R_{k}^{\text {th }}=336 \mathrm{kbps}$.

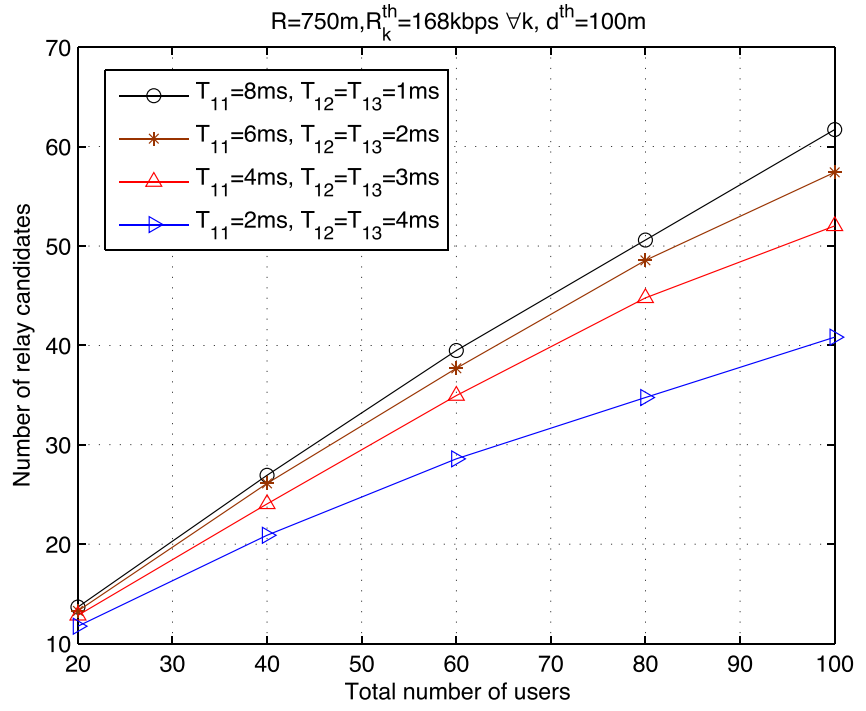

Fig. 9. Number of user-relay candidates for $R_{k}^{\text {th }}=168 \mathrm{kbps}$.

Figs. 9 and 10, respectively. It is illustrated that longer duration of first subframe increases the number of user-relay candidates. However, the advantage of having more user-relays is not reflected on the percentage of satisfied users since there is not enough available subchannels at remaining subframes with duration of $T_{12}$ and $T_{13}$. Higher duration of $T_{11}$ not only means lower duration of $T_{12}$ and $T_{13}$ but also means lower number of resources in these subframes. The users which require the help of the user-relays can not get enough data rate because of the insufficient resources of these subframes and they will not satisfy their threshold data rates. Consequently, the duration of subframes is selected as $T_{11}=4 \mathrm{~ms}$ and $T_{12}=T_{13}=3 \mathrm{~ms}$ while providing further performance results.

We provide the simulation results considering relay selection coverage area radius $d^{\text {th }}$, and users' threshold data rate values $R_{k}^{\text {th }}$ to observe the effects of these parameters on the performance of these four schemes. Firstly, the relay selection coverage area radius $d^{\text {th }}$ is set to $100 \mathrm{~m}$ and $200 \mathrm{~m}$, respectively. The comparison of the

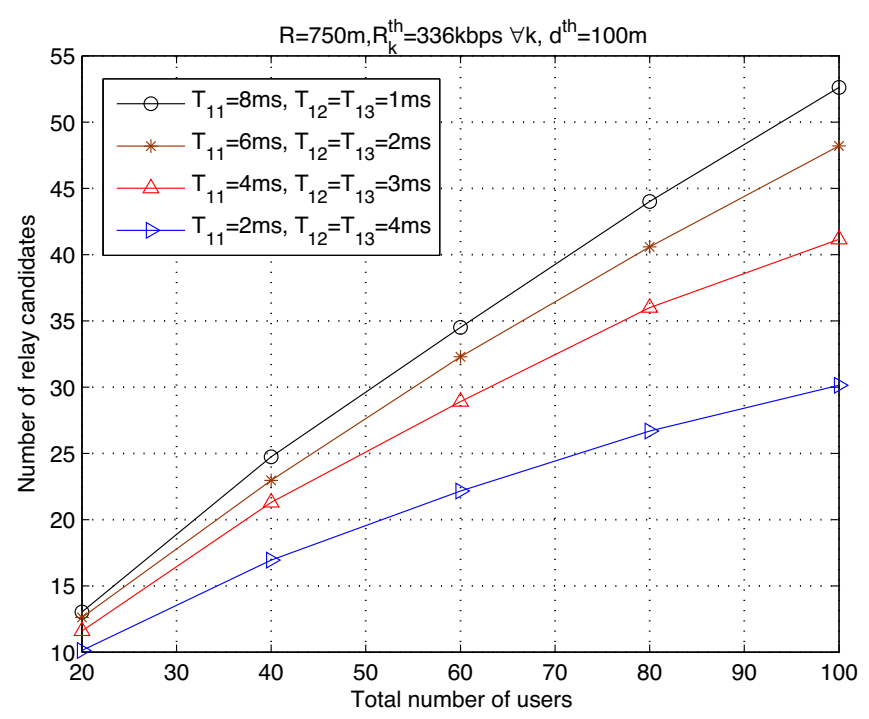

Fig. 10. Number of user-relay candidates for $R_{k}^{\text {th }}=336 \mathrm{kbps}$. 


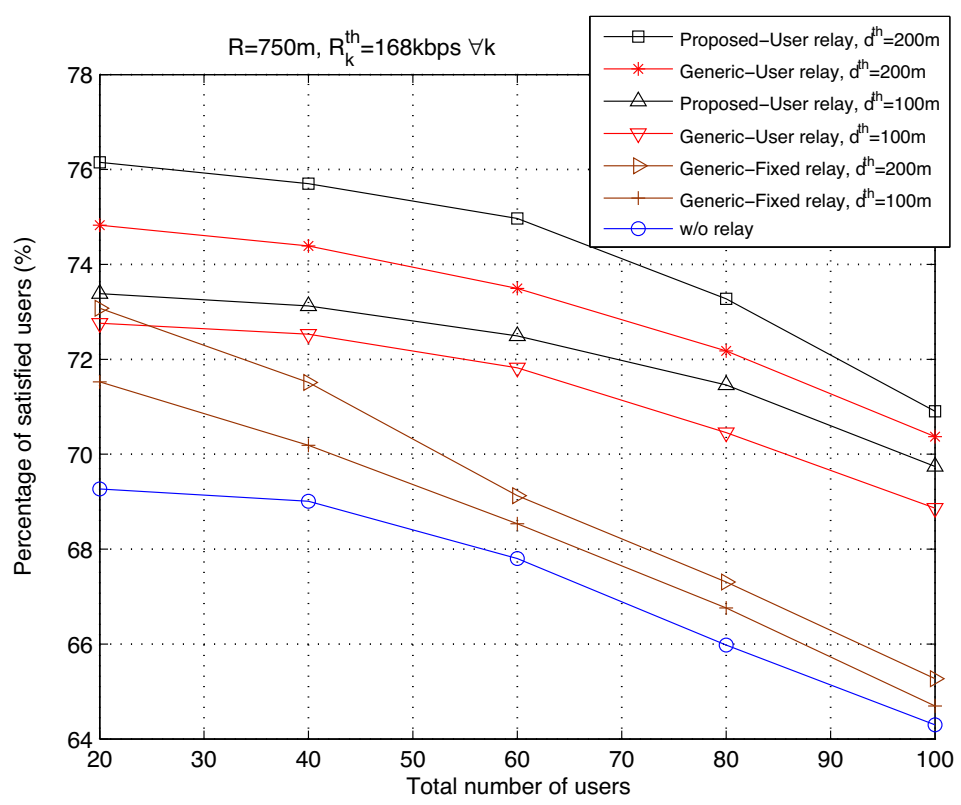

Fig. 11. Comparison of satisfied users' percentage for different $d^{\text {th }}$ values.

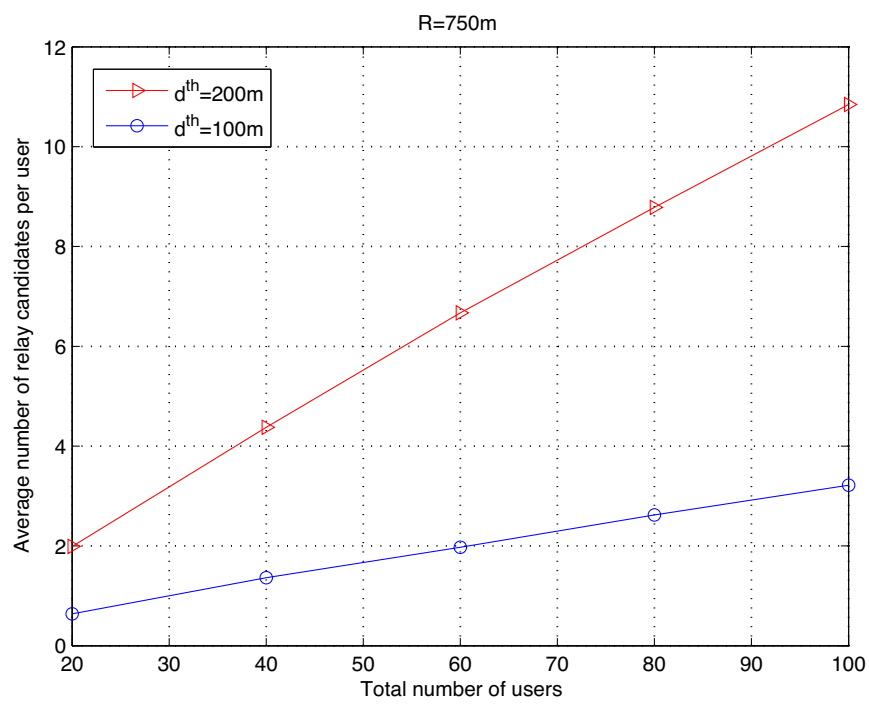

Fig. 12. Average number of relay candidates for each user for different coverage area radius.

schemes in terms of percentage of satisfied users for $R_{k}^{\text {th }}=168 \mathrm{kbps}$ is given in Fig. 11. As it is illustrated, the proposed frame scheme outperforms the non-relaying and fixed relays with generic frame schemes for both radius values. The proposed scheme also gives slightly better performance than the user-relaying scheme with generic frame. In addition to that, the performance on the percentage of satisfied users is improved by increasing the $d^{\text {th }}$ value from $100 \mathrm{~m}$ to $200 \mathrm{~m}$ for all schemes. However, increasing relay selection coverage area radius not only improve the performance on user satisfaction ratio but also increase the signalling overhead. In Fig. 12, the average number of user-relays that remain in the relay selection coverage area is shown to comment on the signalling overhead which depends on the number of relay candidates. In this figure, the number of relay candidates are changing between 1 and 3 for $d^{\text {th }}=100 \mathrm{~m}$ and 2 to 11 for $d^{\text {th }}=200 \mathrm{~m}$ depending on the number of users.

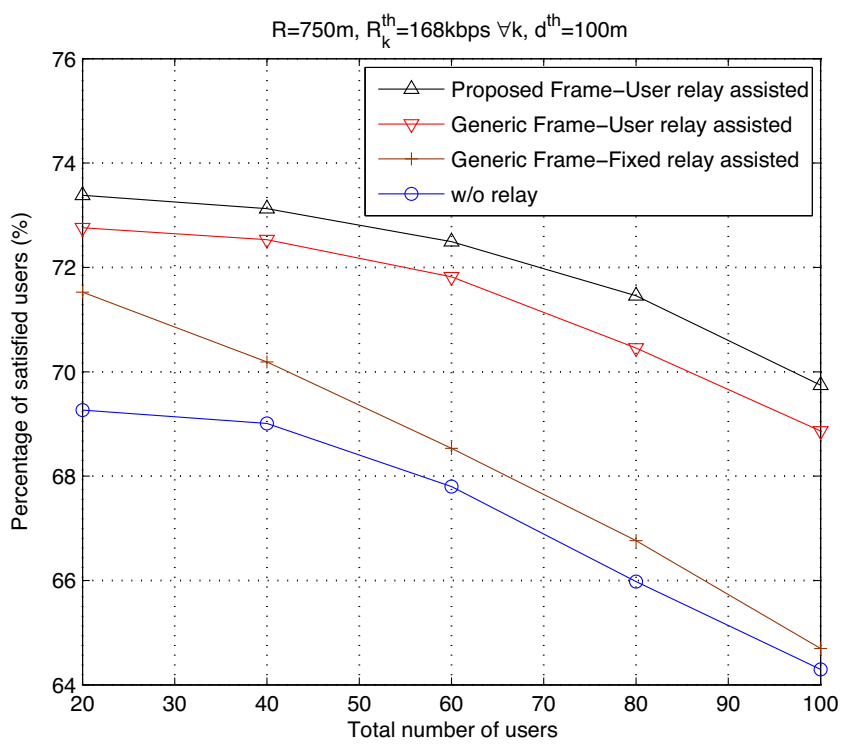

Fig. 13. Percentage of satisfied users for $R_{k}^{\text {th }}=168 \mathrm{kbps}$.

Furthermore, we obtain the comparison results for different threshold data rates of users in order to evaluate the robustness of the proposed frame structure. Figs. 13 and 14 illustrate the percentage of the satisfied users as a function of the number of users in the cell for $R_{k}^{\text {th }}=168 \mathrm{kbps}$ and $R_{k}^{\text {th }}=336 \mathrm{kbps}$, respectively. The relay selection coverage area radius is set to $100 \mathrm{~m}$ in order to decrease the signalling overhead which is especially important for the user-relay assisted networks. The proposed frame structure provides better performance than non-relaying and fixed relay with generic frame schemes for both threshold values. Furthermore, the percentage of satisfied users is enhanced slightly by the proposed frame compared to the generic frame for user-relaying. The proposed scheme eliminates the drawbacks of generic frame structure for user-relay assisted OFDMA networks in which a user acts as a destination and a relay simultaneously, while slightly increasing the percentage of satisfied users. When the number of 


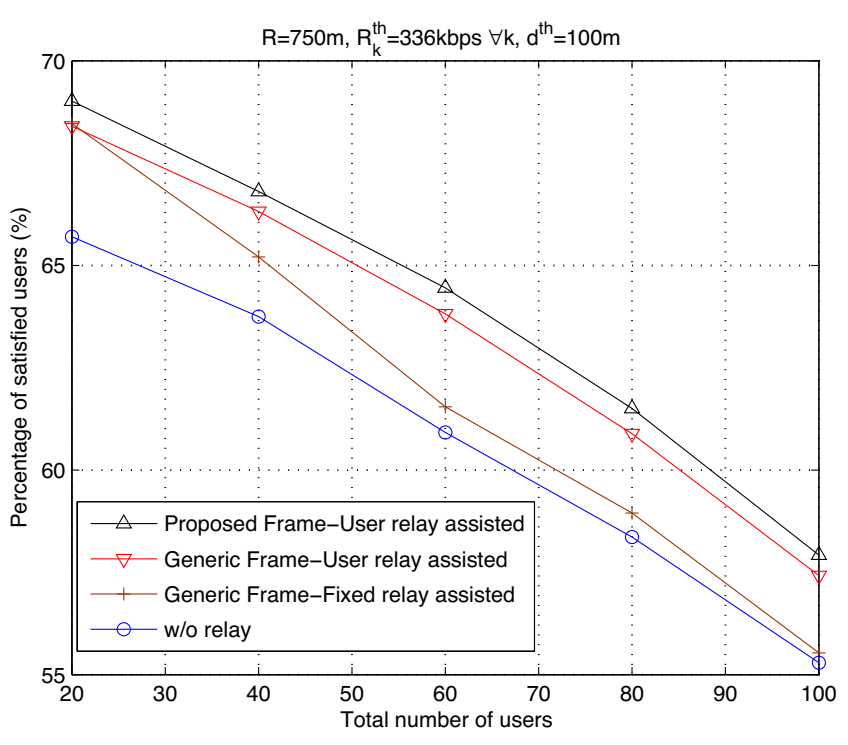

Fig. 14. Percentage of satisfied users for $R_{k}^{\text {th }}=336 \mathrm{kbps}$.

users increases in the cell, the user-relay assisted OFDMA-based networks give much higher performance gain compared to fixed relaying based OFDMA-based networks. The reason is that when the number of users increase in the cell, there will be more choices for user-relay candidates to optimize the system performance. The four schemes are compared in terms of sum rate of the users in Fig. 15 to evaluate the whole system performance. The proposed scheme outperforms the $w / 0$ relay and the fixed-relay assisted schemes with generic frame and also it increases the overall rate slightly compared to the user-relay assisted scheme with generic frame.

Finally, the computational complexity of the four schemes are compared by measuring the execution time required by each of them. Table 2 represents the average execution time needed by each scheme for $K=100$ users. The results given in Table 2 is obtained by using MATLAB R2011b on a PC with Core 2 Duo processor operating with a clock $3 \mathrm{GHz}$. As expected, the scheme that use no relays has the lowest computational complexity. The computational complexity of the fixed relay assisted scheme with generic frame is also lower than the computational complexity of the
Table 2

Average execution time (s) for $K=100$.

\begin{tabular}{lllll}
\hline $\begin{array}{l}\text { Number } \\
\text { of users }\end{array}$ & $\begin{array}{l}\text { Proposed } \\
\text { frame user } \\
\text { relay assisted }\end{array}$ & $\begin{array}{l}\text { Generic } \\
\text { frame user } \\
\text { relay assisted }\end{array}$ & $\begin{array}{l}\text { Generic } \\
\text { frame fixed } \\
\text { relay assisted }\end{array}$ & w/o relay \\
\hline 100 & 6.15 & 6.14 & 5.25 & 4.45 \\
\hline
\end{tabular}

user-relay assisted schemes with both generic and proposed frame. The proposed and generic frame structure for user-relay assisted schemes have almost the same computational complexity.

\section{Conclusions}

In this paper, we have proposed a novel relaying frame structure and developed efficient RRM algorithms for user-relay assisted OFDMA-based wireless networks. The proposed frame structure has three phases and the user can act as a relay if it fullfills its data rate requirements. In most of the works which use generic frame structure for user-relays assume that the users not only receive their own data from the BS but also transmit the other users' data simultaneously. However, this assumption may have practical limitations with the current transceiver design therefore the proposed scheme may be much more suitable for practical implementation. The performance evaluations of the proposed frame structure with user-relays have been compared with the existing non-relaying scheme and generic frame with user-relays and fixed relays in terms of percentage of satisfied users. The performance results indicate that the proposed scheme outperforms the non-relaying scheme and fixed relaying with generic frame and it also slightly improves the performance of user-relaying with the generic frame while avoiding to receive and transmit data by users simultaneously without increasing computational complexity. Moreover, the simulation results reveal that the user-relay assisted networks increase the users' satisfaction ratio without the need of fixed relay stations which is expensive and cost inefficient to plan, optimize and maintenance. This gain is more prominent for higher number of users since the number of relays scales with the number of users in user-relay assisted networks.

In this paper, the subframes duration of the proposed frame structure is determined in a heuristic manner. However, this is an optimization problem and as a future work, the subframes duration of the proposed frame will be determined optimally.

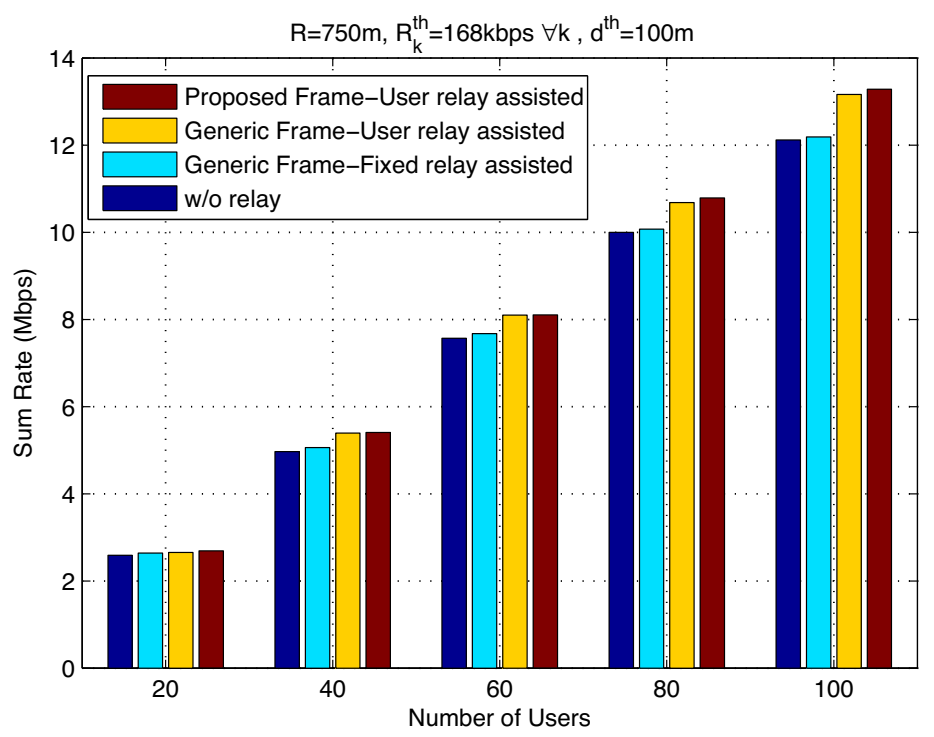

Fig. 15. Sum rate performance for $R_{k}^{\text {th }}=168 \mathrm{kbps}$. 


\section{References}

[1] Andrews JG, Ghosh A, Muhamed R. Fundamentals of WiMAX: understanding broadband wireless networking. Upper Saddle River, NJ: Prentice Hall; 2007. p. $199-220$.

[2] Sendonaris A, Erkip E, Aazhang B. User cooperation diversity - Part I: system description. IEEE Trans Commun 2003;51(11):1927-38.

[3] Sendonaris A, Erkip E, Aazhang B. User cooperation diversity - Part II: implementation aspects and performance analysis. IEEE Trans Commun 2003;51(11):1939-48.

[4] Nosratinia A, Hunter TE, Hedayat A. Cooperative communication in wireless networks. IEEE Commun Mag 2004;42(10):74-80.

[5] Laneman J, Tse DNC, Wornell G. Cooperative diversity in wireless networks: Efficient protocol and outage behavior. IEEE Trans Inform Theory 2004;50(12):3062-80.

[6] Can B, Yanikomeroglu H, Onat FA, Carvalho ED, Yomo H. Efficient cooperative diversity schemes and radio resource allocation for IEEE 802.16j. In: Proc IEEE wireless communications and networking conference (WCNC). 2008. p. $36-41$.

[7] Sreng V, Yanikomeroglu H, Falconer DD. Coverage enhancement through twohop relaying in cellular radio systems. In: Proc IEEE wireless communications and networking conference (WCNC). 2002 March. p. 881-5.

[8] Pabst R, Walke BH, Schultz DC, Herhold P, Yanikomeroglu H, Mukherjee S, et al. Relay-based deployment concepts for wireless and mobile broadband radio. IEEE Commun Mag 2004;42(9):80-9.

[9] Le L, Hossain E. Multihop cellular networks: potential gains, research challenges, and a resource allocation framework. IEEE Commun Mag 2007;45(9): $66-73$.

[10] Kim J, Lee J, Son K, Song S, Chong S. Two-hop opportunistic scheduling in cooperative cellular networks. IEEE Trans Veh Technol 2012:61(9):4194-8.

[11] Yanikomeroglu H. Fixed and mobile relaying technologies for cellular networks. In: Second workshop on applications and services in wireless networks (ASWN). 2002 July. p. 75-81.

[12] Bakaimis B, Lestable T. Connectivity investigation of mobile relays for next generation wireless systems. In: Proc IEEE vehicular technology conference (VTC) - Spring. 2005 May. p. 2192-5.

[13] Nourizadeh H, Nourizadeh S, Tafazolli R. Performance evaluation of cellular networks with mobile and fixed relay station. In: Proc IEEE vehicular technol ogy conference (VTC) - Fall. 2006 September. p. 1-5.

[14] Hoymann C, Wanshi C, Montojo J, Golitschek A, Chrysostomos K, Xiaodong S Relaying operation in 3GPP LTE: challenges and solutions. IEEE Commun Mag 2012;50(2):156-62

[15] Xiao L, Fuja TE, Costello DJ. Mobile relaying: coverage extension and throughput enhancement. IEEE Trans Commun 2010;58(9):2709-17.

[16] Sui Y, Papadogiannis A, Svensson T. The potential of moving relays - a performance analysis. In: Proc IEEE vehicular technology conference (VTC) - Spring. 2012 May. p. 1-5

[17] Tehrani M, Uysal M, Yanikomeroglu H. Device-to-device communication in 5G cellular networks: challenges, solutions, and future directions. IEEE Commun Mag 2014;52(5):86-92.

[18] Chin WH, Fan Z, Haines R. Emerging technologies and research challenges for 5G wireless networks. IEEE Wireless Commun 2014;21(2): 106-12.

[19] Salem M, Adinoyi A, Yanikomeroglu H, Falconer DD. Opportunities and challenges in OFDMA-based cellular relay networks: a radio resource management perspective. IEEE Trans Veh Technol 2010;59(5):2496-510.

[20] Salem M, Adinoyi A, Rahman M, Yanikomeroglu H, Falconer DD, Kim Y, et al. An overview of radio resource management in relay-enhanced OFDMA-based networks. IEEE Commun Surveys Tutorials 2010;12(3):422-38.

[21] Nam W, Chang W, Chung SY, Lee Y. Transmit optimization for relay-based cellular OFDMA systems. In: Proc IEEE international conference on communications (ICC). 2007 June. p. 5714-9.

[22] Huang L, Rong M, Wang L, Xue Y, Schulz E. Resource allocation for OFDMA based relay enhanced cellular networks. In: Proc IEEE vehicular technology conference (VTC) - Spring. 2007 April. p. 3160-4.

[23] Salem M, Adinoyi A, Yanikomeroglu H, Falconer DD, Kim Y. A fair radio resource allocation scheme for ubiquitous high-data-rate coverage in OFDMA based cellular relay networks. In: Proc IEEE global telecommunication conference (GLOBECOM). 2009. p. 1-6.
[24] Wang J, Ma Z, Lv Z, Sheng Y, Xiang W. Fairness-aware resource partition and routing in relay-enhanced orthogonal-frequency-division-multiple-accessing cellular networks. IET Commun 2012;6:2613-20.

[25] Park CW, Lee HJ, Lim JT. Fair semi-distributed resource allocation scheme over relay-enhanced OFDMA networks. IEEE Commun Lett 2012;16(8):1188-91.

[26] Alam MS, Mark JW, Shen X. Relay selection and resource allocation for multi-user cooperative OFDMA networks. IEEE Trans Wireless Commun 2013;12(5):2193-205.

[27] Alazemi HMK, Uddin MF. Fair resource allocation and DF relay selection for multiuser OFDMA-based cooperative networks. Wireless Person Commun 2013;19(6):1485-96.

[28] Oyman O. Opportunistic scheduling and spectrum reuse in relay-based cellular networks. IEEE Trans Wireless Commun 2010;9(3):1074-85.

[29] Choi BG, Bae SJ, Cheon K, Park AS, Chung MY. Relay selection and resource allocation schemes for effective utilization of relay zones in relay-based cellular networks. IEEE Commun Lett 2011;15(4):407-9.

[30] Ding M, Liu S, Luo H, Chen W. MMSE based greedy antenna selection scheme for AF MIMO relay systems. IEEE Signal Process Lett 2010;17(5):433-6.

[31] Clarke P, de Lamare RC. Transmit diversity and relay selection algorithms for multirelay cooperative MIMO systems. IEEE Trans Veh Technol 2012;61(3):1084-98.

[32] Han Z, Himsoon T, Siriwongpairat WP, Liu KJR. Resource allocation for multiuse cooperative OFDM networks: who helps whom and how to cooperate. IEEE Trans Veh Technol 2009;58(5):2378-91.

[33] Shim W, Han Y, Kim S. Fairness-aware resource allocation in a cooperative OFDMA uplink system. IEEE Trans Veh Technol 2010;59(2):932-9.

[34] Ng TCY, Yu W. Joint optimization of relay strategies and resource allocations in cooperative cellular networks. IEEE J Selected Areas Commun 2007:25(2):328-39.

[35] Weng L, Murch RD. Cooperation strategies and resource allocations in multiuser OFDMA systems. IEEE Trans Veh Technol 2009;58(5):2331-42.

[36] Shenghong L, Murch RD. Realizing cooperative multiuser OFDMA systems with subcarrier resource allocation. IEEE Trans Wireless Commun 2013;12(4):1923-35.

[37] Basturk I, Ozbek B, Edemen C, Tan AS, Zeydan E, Ergut S. Radio resource management for OFDMA-based mobile relay enhanced heterogenous cellular networks. In: Proc IEEE vehicular technology conference (VTC) - Spring. 2013 June. p. 1-5.

[38] Basturk I, Ozbek B, Le Ruyet D. Queue aware resource allocation for OFDMAbased mobile relay enhanced networks. In: International symposium on wireless communication systems (ISWCS). 2013 August. p. 1-5.

[39] Bletsas A, Khisti A, Reed D, Lippman A. A simple cooperative diversity method based on network path selection. IEEE J Selected Areas in Commun 2006;24(3):659-72.

[40] Beres E, Adve R. Selection cooperation in multi-source cooperative networks IEEE Trans Wireless Commun 2008:7(1):118-27.

[41] Ikki SS, Ahmed MH. Performance analysis of generalized selection combining for amplify-and-forward cooperative-diversity networks. In: Proc IEEE international conference on communications (ICC). 2009 June. p. 1-6.

[42] Jing Y, Jafarkhani H. Single and multiple relay selection schemes and their achievable diversity order. IEEE Trans Wireless Commun 2009;8(3):1414-23.

[43] Adinoyi A, Fan Y, Yanikomeroglo H, Poor HV, Al-Shaalan F. Performance of selection relaying and cooperative diversity. IEEE Trans Wireless Commun 2009;8(12):5790-5.

[44] Calcev G, Bonta J. OFDMA cellular networks with opportunistic two-hop relays EURASIP J Wireless Commun Networking 2009, http://dx.doi.org/10.1155/ 2009/702659.

[45] Papadogiannis A, Alexandropoulos GC, Burr A, Grace D. Bringing mobile relays for wireless access networks into practice-learning when to relay. IET Commun 2012;6(6):618-27.

[46] Dang W, Tao M, Mu H, Huang J. Subcarrier-pair based resource allocation for cooperative multi-relay OFDM systems. IEEE Trans Wireless Commun 2010;9(5):1640-9.

[47] Chang JM, Hsiao WT, Chen JL, Chao HC. Mobile relay stations navigation-based self-optimization handover mechanism in WiMAX networks. In: Proc ICUT. 2009. p. 1-5.

[48] Sreng V, Yanikomeroglu H, Falconer DD. Relay selection strategies in cellular networks with peer-to-peer relaying. In: Proc IEEE Vehicular Technology Conference (VTC) - Fall. 2003. p. 1949-53. 